\title{
A Comparative Study of 1D Monatomic Fe-chain and 3D Fe-bulk within the Density Functional Theory (DFT)
}

\author{
D. P. Rai ${ }^{1, *}$, Sandeep Chettri ${ }^{2}$, A. Shankar ${ }^{3}$, P. K. Patra ${ }^{4}$, R. K. Thapa ${ }^{2}$ \\ ${ }^{1}$ Department of Physics, Pachhunga University College, Aizawl, 796001, India \\ ${ }^{2}$ Department of Physics, Mizoram University, Aizawl, 796004, India \\ ${ }^{3}$ Department of Physics, University of North Bengal, Darjeeling, 734013, India \\ ${ }^{4}$ Faculty in Centre for Science Education, NEHU Shillong, 794022, India
}

Copyright (C) 2016 by authors, all rights reserved. Authors agree that this article remains permanently

open access under the terms of the Creative Commons Attribution License 4.0 International License

\begin{abstract}
The structural, electronic and magnetic properties of Fe bulk and the monatomic Fe-nano wire has been theoretically investigated within the full potential linearized augmented plane wave (FP-LAPW) based on density functional theory (DFT). This work is a comparative study of Fe-bulk and the 1D Fe chain. A most commonly adopted GGA approximation is use for electron exchange correlation. In our calculation the property of bulk is predicted to be magnetic metal.Though there is no significant band gap at the Fermi level $\left(\mathrm{E}_{F}\right)$ in $\mathrm{Fe}$-wire, whereas the presence of deep valley (Spin up) and a peak(Spin down) at the $\mathrm{E}_{F}$ shows the phenomenon of high antisymmetric density of states (DOS). The presence of high antisymmetric DOS may lead to spin polarization at the $\mathrm{E}_{F}$. Also the two anti-symmetric sharp peaks at two different energies give the exchange splitting. The exchange splitting between the degenerated d-states is dispersed and not much effective in the bulk system but the splitting between $\mathrm{t}_{2 g}$ states is very prominent in Fe-chain which enhanced the magnetic moment.
\end{abstract}

Keywords DFT, GGA, 1D Fe-wire, Magnetism

\section{Introduction}

The magnetic properties of nano particles, thin films and nano wires have recently attracted a lot of attention due to their potential technological applications. It is thus very important to investigate the influence of dimensionality on these properties. In many experimental systems, some atoms are in a bulk-like environment while some others have a very low coordination in a strongly asymmetric environment. As a consequence, the magnetic properties of such systems need to be treated at an atomic level, by studying their electronic structure in the framework of quantum mechanics. In principle, these properties can be determined from $a b$ initio calculations. However, the computer time and storage increase drastically with the number of inequivalent atoms. It is known through theoretical analysis that the one-dimensional atomic array shall not be ferromagnetic in many cases. However several reports revealed that slightly disordered monatomic atom array might have ferromagnetic ground state $[1,2,3]$. From the experimental works the ferromagnetism is confirmed for one-dimensional Co wire which is self assembled on the Pt (997) plane [4]. Before this work several researches were carried out on the ferro-magnetism of nanometer sized structure formed using steps in the (11n) plane $[5,6]$. Since then tremendous researches are made in one dimensional monatomic wires. Among them the ferromagnetism in $\mathrm{Fe}$ monatomic wire formed on $\mathrm{Au}$ (111) plane was confirmed using angle-resolved photo-emission spectroscopy [7] and also the same group reported that the magnetic direction of the Fe monatomic wire self organized on the terrace edge of $\mathrm{Au}$ (788) [8]. In addition Spisak and Hafner carried out ab initio calculation on the Fe monatomic wire on the $\mathrm{Cu}(11 \mathrm{n})$ plane and reported that Fe becomes ferromagnetic and its Curie temperature is between 200 and 300 $\mathrm{K}$ [9]. In recent experimental paper, Mn atomic wire was successfully mounted on the W(111) substrate and the spin polarization at different sites in relation to spin orientation was calculated. The orientation of moment is controlled by an applied magnetic field, the changes of orientation at different sites gives the Dzyaloshinskii-Moriya interaction (DMI effect)[10]. In this work we have carried out $a b$ initio calculation on the 1D monatomic Fe wire and Fe-bulk. We examined the electronic and magnetic properties at their respective dimensions also we tried to analyze the change in the magnetic moment with the change in structural dimensionality.

\section{Model and calculation details}

The model of monatomic Fe-nano wire is formed by the supercell method in which the position of $\mathrm{Fe}$ atom is taken as $(0.5,0.5,0.5)$ and the vacuum of $10 \AA$ As applied along $\mathrm{x}$-axis and $y$-axis in order to discontinue the atomic wave function and to break the crystal symmetry along $\mathrm{x}$ and $\mathrm{y}$ direction. As a result of this the repetition of the atom takes place only in one direction i.e. along the z-axis. The structure thus obtained is $1 \mathrm{D}$ Fe atomic chain (periodic linear chain of iden- 
tical atoms with a single atom per unit cell) with the repetition along z-axis as shown in Fig.1a. The lattice constant (a and $b$ ) of the Fe-wire has been changed to $a=b=10 \AA$. The lattice constant (c) was optimized and the plot of energy versus $\mathrm{c}$ is shown in Fig.1a(top). The optimized lattice constant (c) of Fe-wire is $2.38 \AA$. The Fe-bulk structure has the bcc structure with space group $\operatorname{Im} 3 \mathrm{~m}$ and Wyckoff positions $(0$, 0, 0) as shown in Fig. 1(bottom). Further we choose the experimental lattice constant of $\mathrm{a}=\mathrm{b}=\mathrm{c}=2.84 \AA[11]$ for structural relaxation of the Fe-bulk. The most stable grand state structure was obtained by volume optimization method [see Fig.1a(bottom)]. The volume optimization gives the minimum negative energy. The minimum negative energy value is the ground state energy of a compound which indicates the stability its structure. The optimized lattice constant $(2.85 \AA)$ is used for the calculation of electronic structures adopting the KS-DFT [13] with FP-LAPW method, as implemented in the WIEN2K package [14]. The electron exchange correlation was treated within generalized gradient approximation (GGA) [15]. Non-spherical contributions to the charge density and potential within the muffin tin (MT) spheres are considered up to $1_{\max }=10$ (the highest value of angular momentum functions). The cut-off parameter is $R_{M T} \times K_{\max }$ $=7$ where $K_{\max }$ is the maxi-mum value of the reciprocal lattice vector in the plane wave expansion and $R_{M T}$ is the smallest atomic sphere radii of all atomic spheres. In the interstitial region the charge density and potential are expanded as a Fourier series with wave vectors up to $G_{\max }=12$ a.u. The Monkhorst-Pack grid of dimensions of $6 \times 6 \times 6$ for the bulk and $5 \times 5 \times 32$ for the Fe-nano wire was generated to sample the Brillouin zone. The number of k-points used in the irreducible part of the Brillouin zone is 286. The criterion for the convergence of the self-consistent DFT calculation is $0.0001 \mathrm{Ry}$ in total energy. However the core states are treated relativistically, the semicore states are treated semirelativistically i.e. ignoring the spinorbit $(\mathrm{SO})$ coupling.

\section{Result and discussion}

Iron can be considered an obvious choice to start studying magnetic, and hence spin polarized systems. The LDA fails to determine the correct ground state structure of Fe [16]. In nature, $\mathrm{Fe}$ is found to be body centered cubic and ferromagnetic. According to the all-electron calculations [16], the LDA gives the face centered cubic non-magnetic structure as the most stable but the PBE-GGA predicts the correct structure (ground state stable ferromagnetic structure). The bcc structure is depicted in Fig.1(a). To investigate the effect of each states we repeat the PBE calculations using FP-LAPW method. For this model of exchange-correlation, the bcc ferromagnetic structures were used. The density of states (DOS) calculated from GGA for both bulk and Fe-wire is shown in Fig. 2. The majority contribution in both the systems (bulk and wire) are due to Fe-d states. The total DOS of bulk extended from $-6 \mathrm{eV}$ to $3.0 \mathrm{eV}$ and from $-3.0 \mathrm{eV}$ to $3.0 \mathrm{eV}$ for $1 \mathrm{D} \mathrm{Fe}$ wire. In case of Fe-wire the DOS above $3.0 \mathrm{eV}$ in conduction band is mainly due to the vacuum that was applied to discontinue the atomic wave functions (see Fig. 2(a) red line). The DOS in the valence band for the bulk system is almost symmetric. The anti-symmetric DOS arises due to the d-d hybridization which confirms the ferromagnetic behaviors. In the case of $1 \mathrm{D} \mathrm{Fe}$-wire, one can see the spin DOS is completely anti-symmetric. The sharp and spiky peaks in the DOS (see Fig.2b) in 1D Fe wire represents the discrete energy bands which can be compared with the band structures as shown in Fig. 5(b). In the case of bulk there are no such sharp and spiky peaks to represent the discrete energy levels. The DOS in the bulk materials are dispersed around the Fermi level $\left(\mathrm{E}_{F}\right)$ in both the spin channels (up and down) which predicts its metallic character (see Fig. 5a). On the other hand the low-flat DOS in the spin up channel and the sharp peak around $\mathrm{E}_{F}$ in spin down region is an interesting feature. The two anti-symmetric peaks in the spin up and down region of the $\mathrm{Fe}$-wire is due to the $\mathrm{d}_{e g}$ and $\mathrm{t}_{2 g}$ states, which attributes an exchange splitting. The result of difference in the amplitude of the DOS peaks at $\mathrm{E}_{F}$ gives higher amplitude of spin DOS, which may give an effect like spin polarization which is a typical signature of the half metallic system [17]. Our result of DOS for $1 \mathrm{D} \mathrm{Fe}$-wire is compared with the results of $\mathrm{Fe}$-atomic chain placed on the $\mathrm{Au}$ (111) surface and the result is in good agreement (see Fig. 2a, Ref.[18]). The electron density plot for Fe-bulk and Fe-wire along 100 plane is presented in Fig.3(a,b). The charge distribution on the surface of Fe-wire is predicted by calculating the Fermi surface. The picture of solenoid type Fermi surface is shown in Fig.4(a,b).

The magnetic moments are the results of the asymmetric spin DOS. The magnetic moment of Fe-bulk and wire are analyzed with variation in lattice constant. The result of lattice constant versus magnetic moment are displayed in Fig. 1(b). Our calculated magnetic moment for the bulk system is $2.24 \mu_{B}$ in good agreement with the previous results. The previous results of magnetic moment for the bulk systems are of $2.28 \mu_{B}$ within LSDA [19], $4 \mu_{B}$ for TB-GGA [20] and the experimental magnetic moment of the Fe bulk system is $2.20 \mu_{B}$ [21] similar result was obtained by Starrost et al.[22]. As compared to the bulk system the magnetic moment of 1D system is high. This is due to the reason that Fe-d states exhibit an exchange splitting between the $t_{2 g}$ states (see fig. $2 \mathrm{~b}$ ) with the splitting value around $1.00 \mathrm{eV}$ in $1 \mathrm{D} \mathrm{Fe}$ wire which is missing in Fe-bulk system. The magnetic moment of the monatomic Fe-chain using tight binding local density approximation (TB-LDA) is $3.20 \mu_{B}$. In earlier calculations a magnetic moment of $2.65 \mu_{B}$ was obtained for $2 \mathrm{D} \mathrm{Fe}(1$ $10)$ slab shows [23] which is less than the magnetic moment of $1 \mathrm{D} \mathrm{Fe}$-wire. From the previous DFT calculation it is found that the 6 layers Fe slabs gives a magnetic moment of $3.66 \mu_{B}$, 5 layers Fe slabs give $3.30 \mu_{B}$ and an infinite number of slabs which mimic the bulk gives magnetic moment of around 2.2 $\mu_{B}$ [24]. Our calculated magnetic moment of $1 \mathrm{D}$ monatomic Fe wire is $2.95 \mu_{B}$ which is higher than the value of bulk system but consistent with the previous results as discussed above.

\section{Conclusion}

The main objective behind this work is to study how a reduced dimensionality may modify the electronic and magnetic properties of the ferromagnetic system $(\mathrm{Fe})$. We have noticed that the reducing in structural dimensionality of ferromagnetic system magnetic systems reduces the magnetic moment as well. Nevertheless our result of magnetic moment for the bulk and 1D Fe-wire systems are consistent with the previous experimental results. However, until now we have no idea how this difference in magnetic moment has occurred 

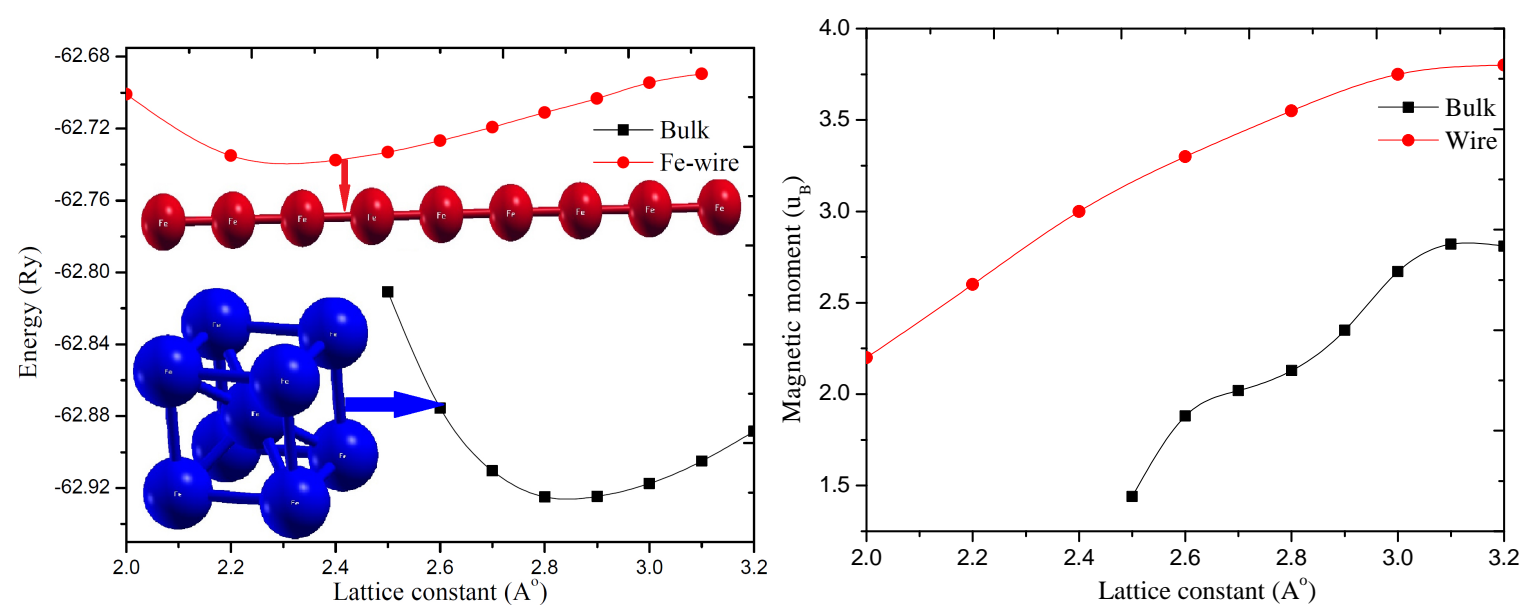

Figure 1. (a) Crystal structure \& Volume optimization curves of 1D Fe-wire and bulk (b) Lattice constant versus magnetic moment
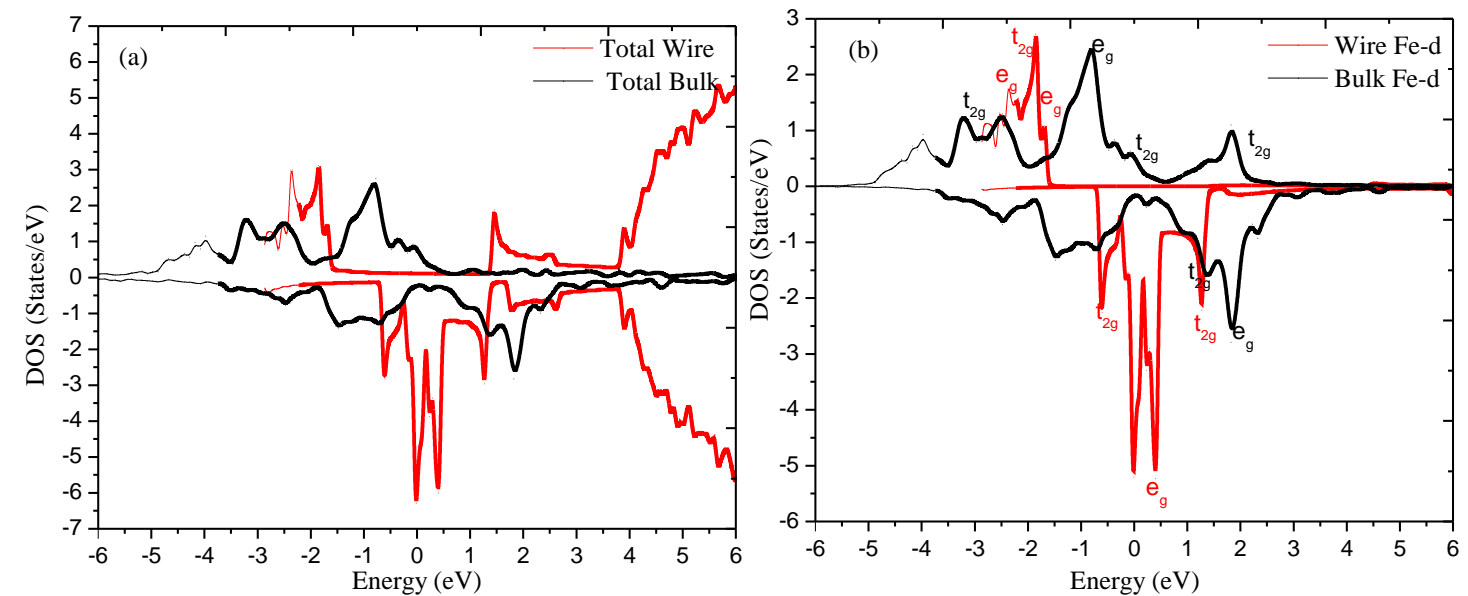

Figure 2. (a) Total DOS (bulk-blue \& Fe chain-red) and (b) Partial DOS of Fe-d (bulk-blue \& Fe chain-red)

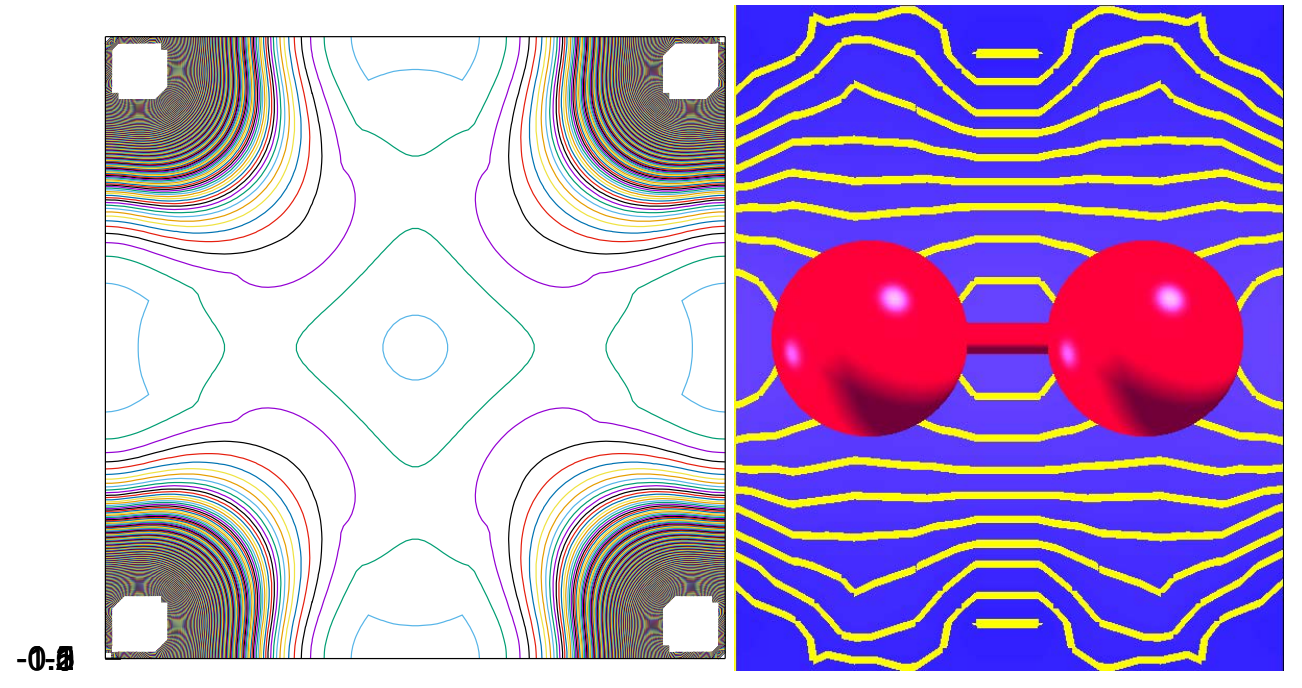

Figure 3. Electron density plot of (a) bulk and (b) wire 

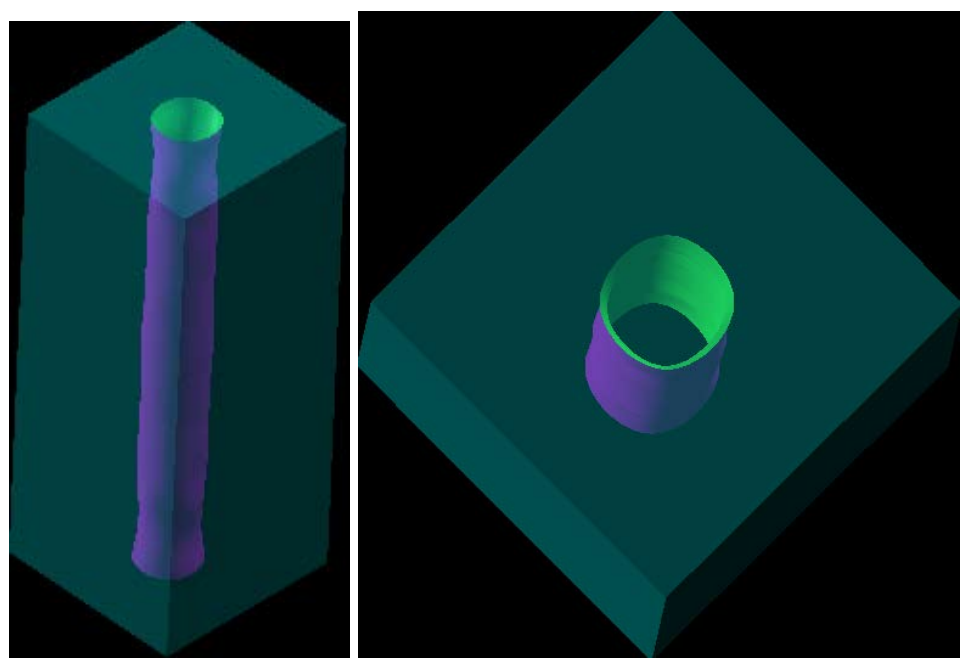

Figure 4. Fermi surface of Fe-wire (a) longitudinal view and (d) cross-section view
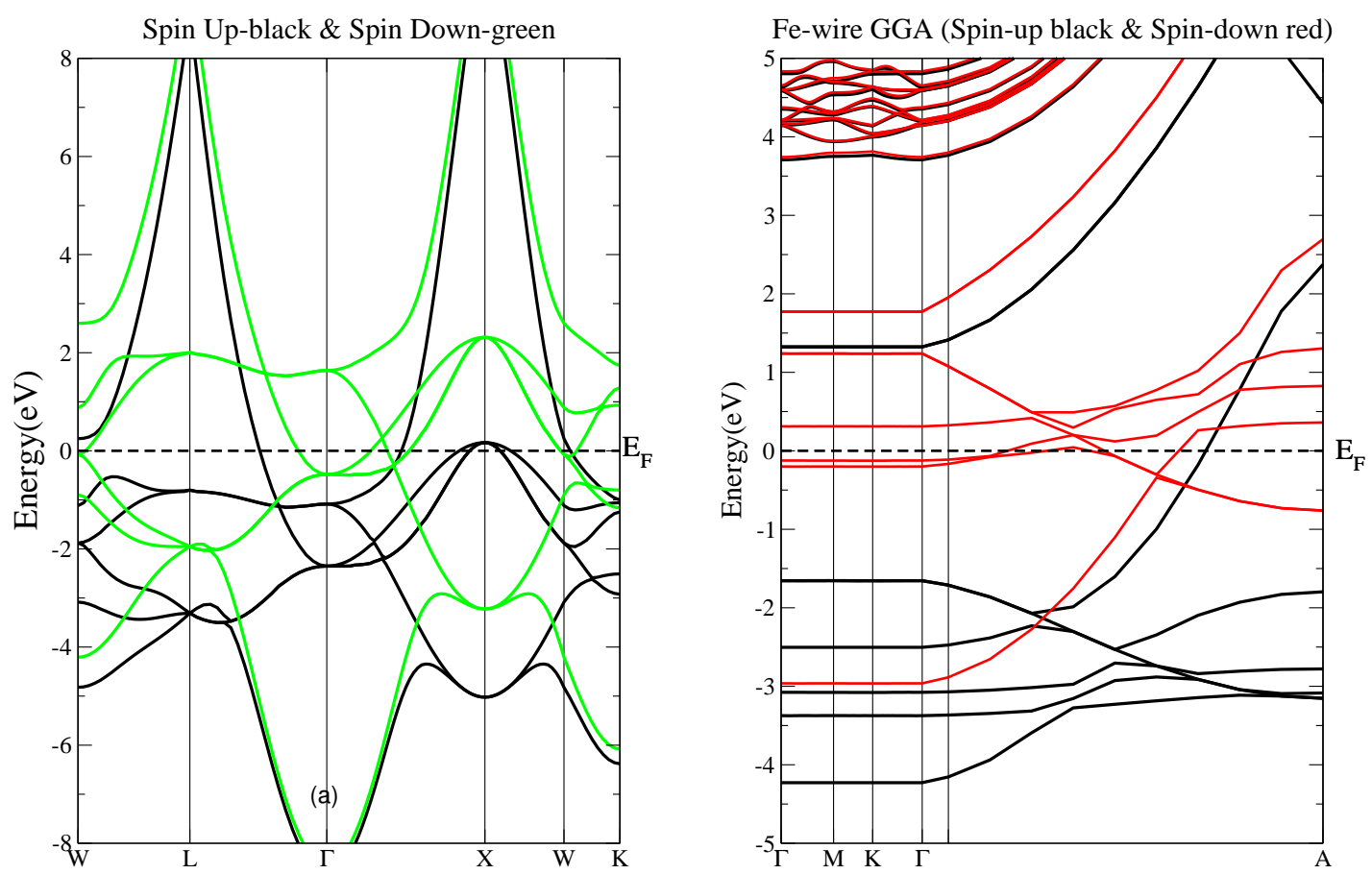

Figure 5. Band structures of (a) Fe-bulk (Spin up-black \& spin down-green) and (b) Fe 1D nano wire (Spin up-black \& spin down-red) 
with the change in the structural dimensionality. We might need further analysis to know the affect of reduced structural dimensionality and its effect on the magnetic moments as well as on its electronic structure.We may intend to use the LSDA+U method to treat the strong correlation of $3 \mathrm{~d}-$ electrons to obtained the most accurate ground state properties.

\section{Acknowledgements}

DPR acknowledges UGC-Start-Up Grant (BSR) (New Delhi, India). SD a grant from DST,New Delhi, India under Dy No. SERB/F/3586/2013-14. Authors thank Dr. Vikas Kasid (Pune University, India) for providing the related information regarding Fe-wire.

\section{REFERENCES}

[1] S. Liang and H. Pang, Europhys. Lett. 32, 173 (1995).

[2] S. Watanabe, M. Ichimura, T. Onogi, Y. Ono, T. Hashizume and Y. Wada, Jpn. J. Appl. Phys. 36, L929 (1997).

[3] S. Daul and M. Noack, Phys. Rev. B 58, 2635 (1998).

[4] P. Gambardella, A. Dallmeyer A, K. Maiti, M. Malagoli, W. Eberhardt, K. Kern and C. Carbone, Nature 416301 (2002).

[5] H. Elmers, J. Hauschild, H. Hoche, U. Gradmann, H. Berthge, D. Heuer and U. Kohler, Phys. Rev. Lett. 73, 898 (1994).

[6] J. Schen, R. Skomski, M. Klaua, H. Jenniches, S. Manoharan and J. Kirschner, Phys. Rev. B 56, 234 (1997).

[7] S. Shiraki, H. Fujisawa, M. Nantoh and M. Kawai, J. Phys. Soc. Jpn. 74, 2033(2005).
[8] H. Fujisawa, S. Shiraki, M. Furukawa, S. Ito, T. Nakamura, T. Muro, M. Nantoh and M. Kawai, Phys. Rev.B 75, 245423 (2007).

[9] D. Spisak and J. Hafner, Phys. Rev. B 65, 235405 (2002).

[10] M. Bode et al., nature 447, 190 (2007).

[11] A. Jephcoat, H. Mao, and P. Bell, Journal of Geophysical Research 91, 4677 (1986).

[12] F.D. Murnaghan Proc. Natl. Acad. Sci. USA 30, 244 (1944).

[13] W. Kohn and L. J. Sham, Phys. Rev. 140, A1133 (1965).

[14] P. Blaha, K. Schwartz, P. Sorantin and S. Trickey, Comp. Phys. Commun59, 399 (1990).

[15] J. Perdew, S. Burke and M. Ernzerhof, Phys. Rev. Lett. 77, 3865 (1996).

[16] E. Blanca, C. Rodriguez, J. Shitu, and D. Novikov, J. Phys.: Condens. Matter 13, 9463 (2001)

[17] I. Galanakis and P. H. Dederichs, Phys. Rev. B 66, 174429 (2002).

[18] T. Yasui and M. Nawate, Journal of Physics: Conference Series 200, 072068 (2010)

[19] V. L. Moruzzi, J. F. Janak, and A. R. Williams, Calculated Electronic Properties of Metals (Pergamon, New York, 1978).

[20] G. Autes et al., J. Phys.: Condens. Matter 1867856813 (2006).

[21] A.H. Morrish, The Physical Principles of Magnetism Wiley: New York,p. 264-272 (1965).

[22] F. Starrost, Phys. Rev. B 64, 235105 (2001).

[23] A.J. Freeman, C.L. Fu, J. Appl. Phys. 61, 3356 (1987).

[24] G. Guzman-Ramirez, J. Robles, A. Vega, F. Aguilera-Granja, J. Chem. Phys. 134, 054101 (2011). 